\title{
Severe gastrointestinal bleeding secondary to duodenal metastasis from a choriocarcinoma
}

\author{
J. Iglesias García ${ }^{1,2}$, E. López García ${ }^{3}$, F. Macías García ${ }^{1,2}$, J. Castro Castro ${ }^{4}$, J. Iglesias Canle,2, \\ C. Beiras ${ }^{5}$, J. Lariño Noia' ${ }^{1,2}$, B. Cigarrán ${ }^{2,6}$, J. Forteza Vila ${ }^{7}$ and J. E. Domínguez Muñoz ${ }^{1,2}$
}

${ }^{1}$ Department of Gastroenterology. ${ }^{2}$ Foundation for Research in Digestive Diseases. ${ }^{3}$ Department of Neurosurgery. University Hospital Xeral-Calde. Lugo. ${ }^{4}$ Department of Neurosurgery and ${ }^{5}$ Department of General Surgery. University Hospital of Santiago de Compostela. A Coruña, Spain. ${ }^{6}$ Department of Internal Medicine. Hospital Virxe da Xunqueira. Cee. A Coruña, Spain. ${ }^{7}$ Department of Pathology. University Hospital of Santiago de Compostela. A Coruña, Spain

\section{CASE REPORT}

A 23-year-old man, operated on March 2004 for a malignant mixed germ-cell tumor in a testicle, was admitted in December 2004 at the Department of Neurosurgery after presenting with a left frontal hematoma, initially related to a left frontal arterio-venous malformation (AVM) (Spetzler grade I). The patient underwent a left fronto-temporal craniotomy, and a total evacuation of the hematoma (Fig. 1). Afterwards, the patient presented with severe gastrointestinal bleeding. A gastroscopy was performed, which identified an ulcerated subepithelial lesion at the $3^{\text {rd }}$ portion of the duodenum with active bleeding (Fig. 2). Hemostasis was achieved after a local injection of adrenalin. After 4 days the patient had signs suggesting rebleeding. At this point, the patient underwent an abdominal arteriogram, which did not show any alterations. A second gastroscopy was then performed, which demonstrated the same duodenal lesion with active bleeding. Hemostasis

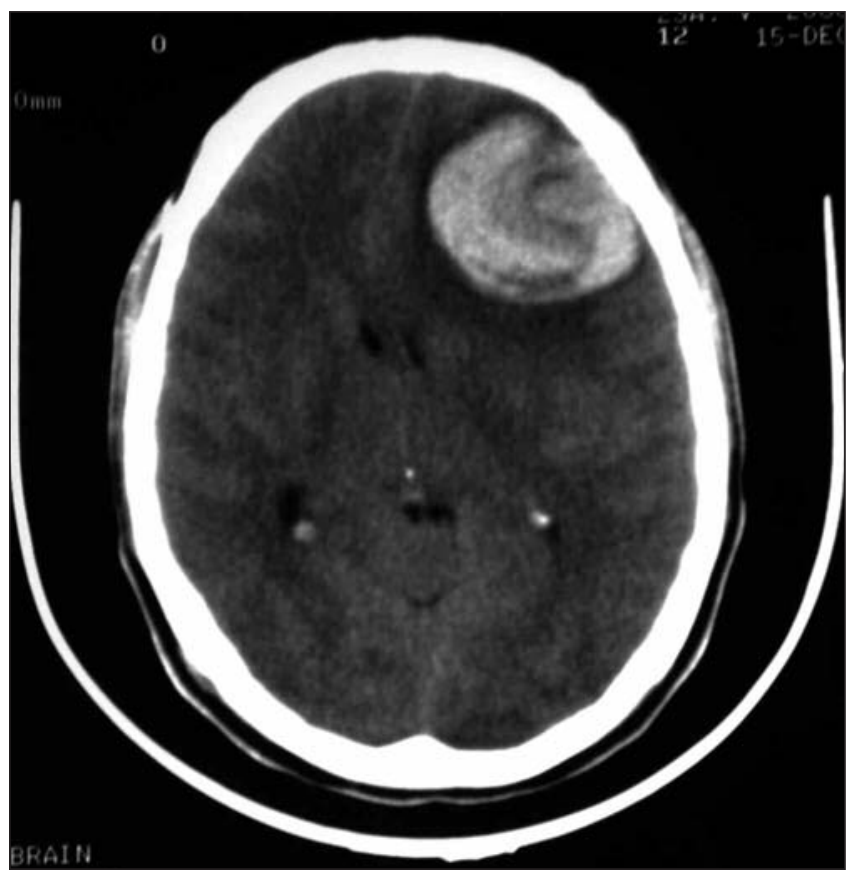

Fig. 1. CT scan showing the left frontal hematoma.

TC craneal mostrando hematoma intraparenquimatoso frontal izquierdo. was again achieved with the injection of adrenalin and argon plasma coagulation. Due to the high risk of rebleeding and the uncertain etiology of the lesion, the patient was scheduled for surgery after endoscopically marking the lesion with hemoclips. The surgical resec-

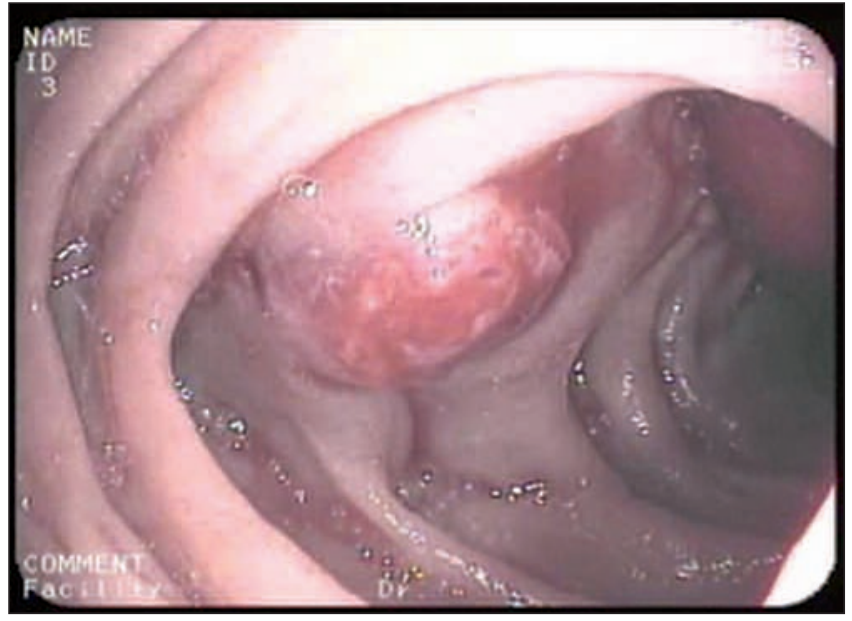

Fig. 2. Endoscopic image of the ulcerated subepithelial lesion located at the third portion of the duodenum.

Imagen endoscópica de lesión subepitelial ulcerada, localizada a nivel de tercera porción duodenal. 
tion was performed with no complications. The patient evolved favorably without signs of rebleeding. Final histology showed an infiltration of the duodenal wall by a choriocarcinoma (syncitiotrophoblastic cells showed positivity for HCG, CK-AE1, CK-CAM 5.2, CK-, and were negative for alkaline phosphatase, vimentin and CKIT). The study was completed with a thoracic and abdominal CT scan. These identified metastatic disease with lesions at pleural, lung, mediastinum, liver, kidney and intraabdominal lymph node levels (Fig. 3). The patient is now under oncological treatment.

\section{DISCUSSION}

Non-gestational choriocarcinoma is a trophoblastic germ-cell tumor that spreads through the lymph and blood from the retroperitoneal space, mediastinum and gonads. Metastatic lesions produce beta-human chorionic gonadotropin. These lesions have hemorrhagic tendency, and thus usually present with bleeding at the implant site. Most characteristic locations include the lung, brain, liver, bones and lymph nodes, affecting the gut in less than $5 \%$ of cases $(1,2)$. In our case, the first clinical manifestation was a left frontal hematoma in the brain.

Related to the gut, the stomach is the organ most commonly affected (mainly fundus and upper body), although there are few cases published in the literature of metastasis affecting the small intestine and colon (2-5). Characteristically, tumor cells cannot pass the capillary barrier of the muscularis mucosae, so implantation takes place in the submucosa. For this reason, gastrointestinal metastasis corresponds to subepithelial lesions and/or ulcerated polypoid masses (3).

Our patient presented with a case of severe gastrointestinal bleeding related to a duodenal subepithelial lesion, a location not previously reported in the literature. In this context, the specific treatment of gastrointestinal metastases remains controversial. There are few communications about surgical resection for isolated lesions $(4,6)$ or of angiographic embolization (5). In our case urgent surgical treatment was needed because of our inability to control bleeding either endoscopically or radiologically. Finally, a histological analysis of the surgical specimen was essential to reach the diagnosis. In this context, the brain hematoma was related to the same pathology.

\section{REFERENCES}

1. Sheinfeld J. Nonseminomatous germ cell tumors of the testis: current concepts and controversias. Urology 1994; $44: 2-14$.

2. Molina Infante J, Beceiro Pedreño I, Ripoll Noiseux C, Marín Jiménez I, González Asanza C, Menchén Fernández-Pacheco P. Gastrointestinal hemorrhage due to metastatic choriocarcinoma with gastric and colonic involvement. Rev Esp Enferm Dig 2004; 96: 77-80.

3. Hsu C, Chen J, Changchien S. Endoscopic features of metastatic tumors in the upper gastrointestinal tract. Endoscopy 1996; $28: 249-53$.

4. Zerbib P, Prieur E, Khory-Helou A, Catala P, Pruvot FR, Chambor JP. Hemorrhagic digestive metastases from testicular choriocarcionoma. Ann Chir 2002; 127: 300-1.

5. Rosenblatt GS, Walsh CJ, Chung S. Metastatic testis tumor presenting as gastrointestinal hemorrhage. J Urol 2000; $164: 1655$.

6. Galloway SW, Yeung EC, Lau JY, Cheng CS. Laparoscopic gastric resection for bleeding metastatic choriocarcinoma. Surg Endosc 2001; 15: 100. 


\title{
Hemorragia digestiva grave por metástasis duodenal de coriocarcinoma
}

\author{
J. Iglesias García ${ }^{1,2}$, E. López García ${ }^{3}$, F. Macías García ${ }^{1,2}$, J. Castro Castro ${ }^{4}$, J. Iglesias Canle ${ }^{1,2}$, \\ C. Beiras ${ }^{5}$, J. Lariño Noia ${ }^{1,2}$, B. Cigarrán ${ }^{2,6}$, J. Forteza Vila ${ }^{7}$ y J. E. Domínguez Muñoz ${ }^{1,2}$
}

${ }^{1}$ Servicio de Aparato Digestivo. ${ }^{2}$ Fundación para la Investigación en Enfermedades del Aparato Digestivo. ${ }^{3}$ Servicio de Neurocirugía. Hospital Xeral-Calde de Lugo. ${ }^{4}$ Servicios de Neurocirugía y ${ }^{5}$ Cirugía General. Hospital Clínico Universitario de Santiago de Compostela. A Coruña. ${ }^{6}$ Servicio de Medicina Interna. Hospital Virxe da Xunqueira de Cee. A Coruña. ${ }^{7}$ Servicio de Anatomía Patológica. Hospital Clínico Universitario de Santiago de Compostela. A Coruña

\section{CASO CLÍNICO}

Varón de 23 años intervenido en marzo de 2004 de un tumor germinal mixto maligno de testículo. En diciembre de 2004 ingresa en el servicio de neurocirugía al presentar un hematoma intraparenquimatoso frontal izquierdo, puesto en relación con una malformación arterio-venosa frontal izquierda (grado I de Spetzler), siendo sometido a craneotomía fronto-temporal izquierda y evacuación del hematoma frontal (Fig. 1). Posteriormente, el paciente presenta una hemorragia digestiva severa. Se realiza una endoscopia digestiva alta identificándose una lesión subepitelial, ulcerada, en $3^{\mathrm{a}}$ porción duodenal (Fig. 2), consiguiéndose hemostasia mediante la inyección local de adrenalina. Tras 4 días estable, el paciente presenta una recidiva del sangrado. Se realiza arteriografía abdominal que no muestra alteraciones; y una nueva endoscopia en la que persiste la misma lesión con sangrado activo. Se realiza tratamiento local mediante adrenalina y electrocoagulación con argón-plasma, consiguiendo hemostasia. Ante el alto riesgo de resangrado y lo incierto de la etiología, se decide tratamiento quirúrgico urgente, marcando endoscópicamente la lesión mediante hemoclip. La resección quirúrgica se realiza sin complicaciones. El paciente evoluciona de forma favorable, sin recidiva del sangrado. El informe histológico fue de infiltración de pared duodenal por coriocarcinoma (positividad de las células de sincitiotrofoblasto para HCG, CKAE1(AE3, CK-CAM 5.2, CK-7; fosfatasa alcalina placentaria, vimentina y C-KIT negativo). Se completó el estudio con TAC toraco-abdominal, identificándose enfermedad metastásica diseminada toraco-abdominal (pleural, pulmonar, mediastínica, hepática, renal y ganglionar intraabdominal) (Fig. 3). En la actualidad el enfermo se encuentra en bajo tratamiento oncológico.

\section{DISCUSIÓN}

El coriocarcinoma no gestacional es un tumor trofloblástico de células germinales que se disemina por vía linfática y sanguínea desde las gónadas, retroperitoneo o mediastino. Las lesiones metastásicas producen gonadotropina coriónica y tienen marcada tendencia hemorrágica, presentándose clínicamente en forma de hemorragias en las zonas de implante. Las localizaciones más características son pulmones, cerebro, hígado, huesos y ganglios linfáticos, mientras que el tracto gastrointestinal se afecta en menos del $5 \%$ de los casos $(1,2)$. En nuestro caso, la manifestación clínica inicial fue un hematoma intraparenquimatoso frontal izquierdo.

Desde el punto de vista gastrointestinal, el estómago es el órgano más afectado (característicamente fundus y cuerpo alto), aunque existen casos publicados de metástasis en intestino delgado y colon (2-5). Las células tumorales no pueden atravesar la barrera capilar de la muscularis mucosae, por lo que se implantan a nivel de la submucosa. Por ello, las lesiones metastásicas gastrointestinales suelen corresponderse a tumoraciones subepiteliales y/o masas polipoideas ulceradas (3).

Nuestro paciente presentó un cuadro de hemorragia digestiva severa en relación con una tumoración submucosa duodenal, localización apenas referida en la literatura. En este contexto, el tratamiento específico de las metástasis gastrointestinales es controvertido. Existen comunicaciones aisladas sobre resección quirúrgica de lesiones aisladas $(4,6)$ o embolización por angiografía (5). En nuestro caso fue preciso un tratamiento quirúrgico urgente, por la imposibilidad de controlar el sangrado ni endoscópica ni radiológicamente. Siendo el análisis de la pieza el que dio el diagnóstico de coriocarcinoma. En este contexto, el hematoma cerebral también se puso en relación con la misma patología. 